INPLASY

PROTOCOL

To cite: Wu et al. Left-side vs. right-side hepatectomy for Hilar Cholangiocarcinoma: A meta-analysis. Inplasy protocol 202130004. doi:

10.37766/inplasy2021.3.0004

Received: 01 March 2021

Published: 02 March 2021

Corresponding author: Jian Wu

21818122@zju.edu.cn

Author Affiliation:

Zhejiang University

Support: [No.81874228].

Review Stage at time of this submission: Data analysis.

Conflicts of interest:

None declared.

\section{Left-side vs. right-side hepatectomy for Hilar Cholangiocarcinoma: A meta-analysis}

$\mathrm{Wu}, \mathrm{WX}^{1} ; \mathrm{Wu}, \mathrm{J}^{2}$.

Review question / Objective: Which side hepatectomy is better for patients. Patients with hilar cholangiocarcinoma. Patients undergone surgery.

Condition being studied: Hilar cholangiocarcinoma; Perihilar cholangiocarcinoma; Left-side hepatectomy; Right-side hepatectomy; Meta-analysis.

Information sources: This meta-analysis followed the Preferred Reporting Items for Systematic Review and MetaAnalyses (PRISMA) statement and the Meta-analysis of Observational Studies in Epidemiology (MOOSE) guidelines. A comprehensive systematic search was performed on PubMed, EMBASE, and Cochrane Library through to February 3, 2020.

INPLASY registration number: This protocol was registered with the International Platform of Registered Systematic Review and Meta-Analysis Protocols (INPLASY) on 02 March 2021 and was last updated on 02 March 2021 (registration number INPLASY202130004).

\section{INTRODUCTION}

Review question / Objective: Which side hepatectomy is better for patients. Patients with hilar cholangiocarcinoma. Patients undergone surgery.

Condition being studied: Hilar cholangiocarcinoma; Perihilar cholangiocarcinoma; Left-side hepatectomy; Right-side hepatectomy; Meta-analysis.

\section{METHODS}

Participant or population: Literature that reported the outcomes of left-side hepatectomy versus right-side hepatectomy in patients with HCCA and met the following criteria were included: (i)randomized controlled trials (RCTs), 
cohort studies or case-control studies; (ii)adult patients with HCCA;(iii) languagefree publication comparing left-side hepatectomy and right-side hepatectomy for HCCA;(iv) include at least one of the following endpoints: overall survival (OS), 1-year survival rate, 3-year survival rate,5year survival rate, operating time, Ro resection rate, postoperative morbidity, PHLF, procedure-related mortality. Exclusion criteria include the following:(i) study design type without explicit accountability;(ii) patients with intrahepatic cholangi o c a r cinoma, dista I cholangiocarcinoma, and gallbladder carcinoma; (iii) no controls;(iv) duplicates; (v) unable to extract valid outcome data from the literature;(vi) conference, editorials, reviews, case reports, commentaries, letters, research involving animal experiments, cohorts wi.

Intervention: Which side of surgery is better.

Comparator: Left-side VS right-side, they were comparator of each other.

Study designs to be included: Literature that reported the outcomes of left-side hepatectomy versus right-side hepatectomy in patients with HCCA and met the following criteria were included: (i)randomized controlled trials (RCTs), cohort studies or case-control studies; (ii)adult patients with HCCA;(iii) languagefree publication comparing left-side hepatectomy and right-side hepatectomy for HCCA;(iv) include at least one of the following endpoints: overall survival (OS), 1-year.

Eligibility criteria: Literature that reported the outcomes of left-side hepatectomy versus right-side hepatectomy in patients with HCCA and met the following criteria were included:(i)randomized controlled trials (RCTs), cohort studies or case-control studies;(ii)adult patients with HCCA;(iii) language-free publication comparing leftside hepatectomy and right-side hepatectomy for HCCA;(iv) include at least one of the following endpoints: overall survival (OS), 1-year survival rate, 3-year survival rate,5-year survival rate, operating time, $\mathrm{RO}$ resection rate, postoperative morbidity, PHLF, procedure-related mortality. Exclusion criteria include the following:(i) study design type without explicit accountability;(ii) patients with intrahepatic cholangiocarcinoma, distal cholangiocarcinoma, and gallbladder carcinoma;(iii) no controls;(iv) duplicates;(v) unable to extract valid outcome data from the literature;(vi) conference, editorials, reviews, case reports, commentaries, letters, research involving animal experiments, cohorts with fewer than 10 cases and when full text was not available.

Information sources: This meta-analysis followed the Preferred Reporting Items for Systematic Review and Meta-Analyses (PRISMA) statement and the Meta-analysis of Observational Studies in Epidemiology (MOOSE) guidelines. A comprehensive systematic search was performed on PubMed, EMBASE, and Cochrane Library through to February 3, 2020.

Main outcome(s): Which is the better choose for HCCA patients.

\section{Additional outcome(s): None.}

Quality assessment / Risk of bias analysis: The literature quality evaluation method adopts the QUADAS diagnostic test quality evaluation form, which is evaluated independently by two participants. If the conclusions are inconsistent, the final decision will be made by the third-party experts.

Strategy of data synthesis: This metaanalysis followed the Preferred Reporting Items for Systematic Review and MetaAnalyses (PRISMA) statement and the Meta-analysis of Observational Studies in Epidemiology (MOOSE) guidelines. A comprehensive systematic search was performed on PubMed, EMBASE, and Cochrane Library through to February 3, 2020. The search strategy was to combine key words including "hilar cholangiocarcinoma", "klatskin tumor", "left-side hepatectomy" and "right-side hepatectomy" into various combinations. 
To identify more relevant literature, manual search was performed on references of all included literature. Restrictions were not placed on any point of the search. In addition, the search process was completed independently by two authors (Wenxuan Wu and Qiyang Cheng), and the disagreement reached consensus through discussion.

Subgroup analysis: Region; Publication year.

Sensitivity analysis: Change the inclusion criteria (especially the controversial studies), exclude low-quality studies, use different statistical methods/models to analyze the same data, etc.

Language: English.

Country(ies) involved: China.

Keywords: Hilar cholangiocarcinoma; Perihilar cholangiocarcinoma; Left-side hepatectomy; Right-side hepatectomy; Meta-analysis.

Contributions of each author:

Author 1 - Wenxuan Wu.

Author 2 - Jian Wu. 